\title{
Feasibility of upright patient positioning and intubation success rates At two academic EDs
}

Joseph S. Turner, Timothy J. Ellender, Enola R. Okonkwo, Tyler M. Stepsis, Andrew C. Stevens, Erik G. Sembroski, Christopher S. Eddy, Anthony J. Perkins, Dylan D. Cooper

\section{Objectives:}

Endotracheal intubation is most commonly taught and performed in the supine position. Recent literature suggests that elevating the patient's head to a more upright position may decrease peri-intubation complications. However, there is little data on the feasibility of upright intubation in the emergency department. The goal of this study was to measure the success rate of emergency medicine residents performing intubation in supine and non-supine, including upright positions.

\section{Methods:}

This was a prospective observational study. Residents performing intubation recorded the angle of the head of the bed. The number of attempts required for successful intubation was recorded by faculty and respiratory therapists. The primary outcome of first past success was calculated with respect to three groups: 0-10 degrees (supine), 11-44 degrees (inclined), and $\geq 45$ degrees (upright); first past success was also analyzed in 5 degree angle increments.

\section{Results:}

A total of 231 intubations performed by 58 residents were analyzed. First pass success was $65.8 \%$ for the supine group, $77.9 \%$ for the inclined group, and $85.6 \%$ for the upright group $(p=$ 0.024). For every 5 degree increase in angle, there was increased likelihood of first pass success $(A O R=1.11 ; 95 \% \mathrm{Cl}=1.01-1.22, \mathrm{p}=0.043)$.

\section{Conclusions:}

In our study emergency medicine residents had a high rate of success intubating in the upright position. While this does not demonstrate causation, it correlates with recent literature challenging the traditional supine approach to intubation and indicates that further investigation into optimal positioning during emergency department intubations is warranted.

Keywords: upright, intubation, laryngoscopy

This is the author's manuscript of the article published in final edited form as:

Turner, J. S., Ellender, T. J., Okonkwo, E. R., Stepsis, T. M., Stevens, A. C., Sembroski, E. G., ... \& Cooper, D. D. (2017). Feasibility of upright patient positioning and intubation success rates at two academic emergency departments. The American Journal of Emergency Medicine, 35(7), 986-992. https://doi.org/10.1016/j.ajem.2017.02.011 


\section{Introduction}

Endotracheal intubation via direct laryngoscopy has been an important procedure in medicine since the late $19^{\text {th }}$ century [1-3]. While commonly performed in intensive care units (ICUs), emergency departments (EDs), and even procedural suites, much of what we know about endotracheal intubation comes from anesthesiology experience in the operating room $(\mathrm{OR})$ and much of the practiced technique is historically driven by operative management. Most commonly, this involves supine patient positioning. Furthermore, while there have been significant advances in technology, including the advent of video laryngoscopy, many aspects of the procedure have remained unchanged for more than a century.

Endotracheal intubation in the ED, often done emergently, in contrast to the controlled context of the OR, has a higher incidence of complications (e.g., hypoxemia, aspiration, and hypotension) and difficult intubation [4-11]. Increasingly reports of "bundled interventions", "process control" of induction sequence, and even patient positioning have been levied as means to reduce complications associated with emergent endotracheal intubation [6, 12-14]. Head-elevated positioning has been shown to improve pre-oxygenation in both obese and nonobese patients [14-17], improve glottic view [18], and reduce complications of intubation [14]. However, data regarding the effect of patient positioning on success rates of emergent endotracheal intubation in an ED environment are lacking.

The goal of this study was to measure the success rate of emergency medicine (EM) residents performing intubation in supine and non-supine, including upright positions. We also sought to measure provider satisfaction and complication rates with endotracheal intubation in an upright position. 


\section{Methods}

\subsection{Study Design and Setting}

This was a prospective observational study conducted at two academic teaching hospitals affiliated with the University Emergency Medicine Residency program. The Hospital is a county hospital with approximately 100,000 patient visits annually. Hospital is a tertiary referral center, also with approximately 100,000 patient visits annually. Data collection occurred from July 17, 2014 July 16,2015 . The study was approved by the University Institutional Review Board.

\subsection{Population}

Prior to initiation of the study, the concept of intubation with the head of the bed elevated to 45 degrees was introduced to residents and faculty at department-wide meetings. A video demonstration was sent to the entire department. Residents participated in a brief practice session in which they intubated a high-fidelity adult mannequin (Laerdal SimMan 3G Stavanger, Norway) in the 45 degree position (Figure 1). Residents who would be performing intubations and supervising faculty were invited to participate in the study on a voluntary basis. All participating residents and supervising faculty signed informed consent statements and attested to watching the demonstration video.

Intubation events that were eligible for data collection included adult medical intubations performed at participating hospitals in which the intubating resident and supervising faculty both consented to study participation. Pediatric, obstetric, and trauma patients were excluded.

\subsection{Study Protocol}

Study packets were attached to airway trays in the participating EDs. Each packet contained a tool for measuring the angle of the head of the bed, faculty and resident preintubation surveys, a stopwatch, a sheet for the nurse to record the procedure time, and post- 
intubation surveys for the resident, faculty, and respiratory therapist (RT). Pre-intubation surveys for the resident and faculty contained a single question which asked about anticipated difficulty of intubation based on overall clinical gestalt on a three point scale: easy, intermediate, or difficult (Table 1).

Residents were asked to set the head of the patient's bed to a position of their choosing. They were instructed to choose without regard to anticipated difficulty of intubation, and to vary position throughout the course of the study. Prior to intubation, if time allowed, residents were asked to use the tool to measure and record the angle of the head of the bed with respect to the floor and fill out the pre-intubation surveys. If the urgency of the procedure did not allow recording of the angle prior to the intubation attempt, they were asked to record it immediately after the procedure. Following the procedure, residents, faculty, and RTs were asked to fill out the post-intubation surveys. Resident surveys included questions regarding angle of intubation, whether position was changed during the procedure, type of blade used, best Cormack-Lehane $(\mathrm{CL})$ view obtained during the procedure, best Percent of Glottic Opening score obtained during procedure, and satisfaction on a three point scale with patient positioning (not satisfied, somewhat satisfied, very satisfied). Faculty and RT post-intubation surveys were modified versions of airway evaluation forms already used by the residency program. Data that were extracted from these cards included number of attempts needed for successful intubation, starting and lowest oxygen saturation, and presence of adverse events (esophageal intubation or cardiac arrest) (Table 1).

Additional data including patient demographics and 5-day outcomes were obtained through chart review.

\subsection{Outcome Measures}

The primary outcome of interest was successful endotracheal intubation on first attempt. An attempt was defined as the act of placing the laryngoscope blade in the patient's oropharynx. At the beginning of the study residents, faculty, and RTs were educated on this definition. Periodic pre-planned reminders of this definition were sent throughout the study duration. In cases in which the faculty and RT recorded a different number of attempts, parties 
were contacted and after discussion to determine what had happened, a consensus on number of attempts was reached. If consensus could not be reached after discussion, data for that intubation were excluded from final analysis.

Secondary outcomes included overall success rate of endotracheal intubation, time required for intubation, esophageal intubation, cardiac arrest within 30 minutes of the intubation attempt, decrease in oxygen saturation during the procedure, best Cormack-Lehane view obtained during the procedure, best Percent of Glottic Opening (POGO) score obtained during the procedure, resident satisfaction with patient positioning, death in the ED, death within 5 days of intubation, and new pneumonia developing within 5 days of intubation.

Total procedure time was measured by a nurse with a stopwatch. The timer was started when the resident first began to insert the laryngoscope blade into the mouth and was stopped when either color change on colormetric end-tidal $\mathrm{CO}_{2}$ detector or bilateral breath sounds was announced, whichever came first. Decrease in oxygen saturation was calculated as the difference between starting saturation and lowest saturation as recorded by faculty and RT. In cases where two different numbers were recorded, the greater difference was used.

Death within 5 days was analyzed according to 3 possible outcomes: no death, death due to withdrawal of care, or death despite continued medical care. New pneumonia within 5 days was also analyzed according to 3 possible outcomes: no pneumonia, pneumonia occurring prior to intubation attempt, or pneumonia developed subsequent to the intubation attempt. Diagnosis of pneumonia was made by reviewing chest radiographs in the days following the intubation attempt and (when available) prior to the intubation attempt, as well as reviewing ICU charts for a diagnosis of pneumonia. In cases of new infiltrate, ED and ICU charts were reviewed for documentation of suspected aspiration prior to the intubation attempt. When clear documentation of such was determined by consensus of study investigators, the outcome was considered a pneumonia occurring prior to intubation attempt. All other pneumonias not present on a pre-procedure radiograph were considered new pneumonias.

\subsection{Data Analysis}


Since gaps in data collection were anticipated due to the difficulty collecting information during an emergent procedure, investigators defined at the beginning of the study the data required for the intubation encounter to be included in final analysis. This included patient medical record number, the angle of head position, and number of attempts as recorded by either faculty or RT.

Patient position was divided into three groups for data analysis: 0-10 degrees ("supine" group), 11-44\% (“inclined" group), and $\geq 45$ degrees ("upright" group). We used chi-square tests and analysis of variance (ANOVA) models to determine if patient characteristics differed by the three position groups. When testing the association between patient position and other characteristics with the primary and secondary outcomes, we used mixed effects models (linear and logistic). The mixed effect models contained fixed effects for patient characteristics and a random effect for resident to account for the clustering of patients intubated by the same resident. We modeled the effect of position (supine, inclined, upright) two different ways, using position as either a classification variable or ordinal variable. The ORs from both sets of models were similar so we only report the model using the ordinal classification. Additionally, we ran mixed-effect logistic regression models using degrees increase in head position in place of the 3 defined groups. Several variables were recoded for mixed effects logistic regression analysis due to either small cell sizes or extremely skewed data. Satisfaction was recoded as very satisfied versus not satisfied/somewhat satisfied and CL view was recoded as I versus II, III, and IV. Saturation decrease was recoded to any saturation decrease versus no saturation decrease since over $50 \%$ of cases had no saturation decrease. The POGO score was recoded as a score of 100 versus a score less than 100 since $60 \%$ of cases had a POGO score of 100 .

\section{Results}

\subsection{Characteristics of Study Subjects}

A total of 67 residents consented to participate in the study and 58 of these residents submitted data. Participating residents performed a range of 1-12 intubations. There were 38 
residents that submitted three or more intubations, and 31 of these residents submitted intubations in multiple positions. Residents were supervised by 60 different consenting faculty. There were 253 data packets submitted over the course of the study. Of these, 15 were excluded because they were missing the predefined required data, and 4 were excluded because they met exclusion criteria (all 4 were trauma patients). One entry was determined to be a duplicate and one entry was excluded because the faculty performed the intubation rather than the resident. Of the remaining 232 intubations, 164 reported data on first past success from both the faculty and the RT, while 68 included only one data point on first-pass success. For 8 intubations there were discrepancies between faculty and RT on whether there was firstpass success; 7 of these discrepancies were resolved by discussion with the involved parties, while in 1 case the discrepancy could not be resolved and the data was therefore excluded. This left 231 intubations for final data analysis (Figure 2). For the 7 discrepancies in which a resolution was reached, 2 were determined to have succeeded on first pass while 5 were determined to have required multiple attempts. Study investigators were the supervising faculty in 36 of the intubations ( 8 supine, 11 inclined, and 17 upright).

\subsection{Main Results}

Residents intubated 38 patients in the supine position (16\%), 68 in the inclined position (29\%), and 125 in the upright position (54\%). Distribution of the angle of the head of the bed can be found in Figure 3. Patient demographics and relevant information about the intubations can be found in Table 2. There were significant differences between groups with respect to indication for intubation, sedative used, and paralytic used. On further analysis, these measures were not associated with likelihood of first-pass success. Additionally, 5 patients in the supine group were undergoing active cardiopulmonary resuscitation (CPR) at the time of intubation, compared with 1 patient in the inclined group and 0 patients in the upright group. There were no significant differences between groups in terms of gender of the patient, resident level, blade used, bougie use, predicted difficulty from the resident, predicted difficulty from the faculty, preceding hypoxia, comorbidities, home oxygen use, patient age, patient weight, or patient body mass index (BMI). 
Results for primary and secondary outcomes are presented in Table 3. For the primary outcome, first pass success rates were higher in the upright group than in the supine and inclined groups, and the difference was statistically significant. When adjusting for other variables including resident experience level, patient age, patient BMI, patient gender, and use of direct versus video laryngoscopy, this difference remained statistically significant. Specifically, for each position increase (supine to inclined, inclined to upright), there was an increased odds of first pass success (AOR=1.75, 95\% Cl = 1.04-2.95) (Table 4). For each 5 degree increase in head position, there was a statistically significant increase in the odds of first pass success $(A O R=1.11,95 \% \mathrm{Cl}=1.01-1.24)$. Resident level was significantly associated with likelihood of first pass success, with more experienced residents having higher rates of first pass success. Patient gender was also associated with first pass success, with male patients being more difficult to intubate than female patients (OR $0.37,95 \% \mathrm{Cl} 0.16-0.87$ ). There was a trend toward increased likelihood of success with video laryngoscopy compared with direct laryngoscopy, though this did not reach statistical significance.

There were 6 intubations performed during ongoing CPR (5 supine and 1 inclined). Excluding these 6 cases, position was still associated with first pass success $(p=0.034)$. However, after excluding these cases and subsequently adjusting for demographics, the first pass success was only marginally different by position $(p=0.062)$.

In terms of secondary outcomes, post-intubation cardiac arrest within 30 minutes of the procedure occurred more often in the supine group compared with the inclined and upright groups $(p=0.006)$. There was no significant difference in rates of esophageal intubation, view obtained, 5-day mortality, development of new pneumonia, oxygen saturation decrease, or time to intubation with respect to position. A higher percentage of residents reported being very satisfied with the upright position compared to the supine and inclined positions, though this did not meet statistical significance.

For secondary outcomes, increased BMI $(p=0.031)$, pre-procedure hypoxia $(p=0.034)$, and decreased resident experience $(p=0.051)$ were associated with significantly increased levels of desaturation. Video laryngoscopy $(p=0.057)$ and female patient gender $(p=0.039)$ were associated with improved Cormack-Lehane view, and video laryngoscopy $(p=0.061)$ was 
marginally associated with improved POGO score. Third year residents were more likely to be satisfied with the procedure compared to second or first year residents $(p=0.032)$. There were no statistically significant differences in 5-day mortality or rates of new pneumonia.

\section{Discussion}

In our study emergency medicine residents intubating patients with the head of the bed elevated to 45 degrees or higher had a high rate of first past success and high rates of satisfaction with patient positioning. Importantly, residents received only very brief training and practice with the technique prior to participating in the study. It is possible that with additional training and experience, the rate of success would have been higher.

There are a number of potential advantages to intubating with the head of the bed in an upright position. Khandelwal et al. found that intubating with the head of the bed elevated above 30 degrees was associated with decreased peri-intubation complications, in particular decreased rates of hypoxemia and aspiration [14]. Upright positioning in the pre-oxygenation period has been associated with longer times to desaturation [15-17]. Standard care in patients with intracranial hemorrhage and elevated intracranial pressure is to keep the head of the bed elevated, and one could hypothesize a benefit to maintaining this position throughout the intubation procedure. Most importantly, emerging evidence suggests that head-elevated positioning may be associated with improved glottic views in both simulated [19] and OR environments [18], suggesting that this technique has the potential to help facilitate successful endotracheal intubation. This is important as first pass success is associated with lower rates of adverse events than when multiple attempts are needed [20].

Consequently, there will likely be increasing use of upright positioning in a variety of care settings. To our knowledge, our study is the first to report on the feasibility of this technique in the ED. We found upright positioning had higher rates of first past success compared with the traditional supine position. The observational nature of our study 
introduces selection bias and a number of potential confounders, limiting what conclusions may be drawn.

We attempted to account for selection bias by collecting data on patient characteristics and procedure elements that could influence intubation difficulty. We also compared faculty and resident pre-intubation predictions of difficulty of the procedure. The groups were similar with respect to anticipated difficulty of intubation, as well as most patient-specific and procedure-specific variables. When differences between the groups did exist, such as sedative and paralytic use, the variables did not have an association with first past success. Furthermore, we used logistic regression to adjust for other variables that potentially could influence success and the results for the primary outcome remained statistically significant.

Nevertheless, there are potentially other important confounders that we did not account for, and it is possible that when residents chose the position they were motivated by other variables that could influence likelihood of success but were not captured in our data. In particular, while it did not reach statistical significance, a larger percentage of patients in the supine group were intubated for cardiovascular dysfunction and potentially were more unstable. Additionally, there were 5 cases of ongoing CPR in the supine group and none in the upright group. When these cases were removed from analysis there was still a statistically significant association between position and first pass success, though this association lost statistical significance when also adjusting for additional demographic factors.

Another limitation of our study is that all eligible intubations were not captured. The institutions involved in the study do not have a central mechanism for tracking data on all emergency department intubations so we do not know how many intubations were missed. Based on resident procedure tracking, we estimate that we captured approximately half of all eligible intubations. It is possible that intubations that were missed differed in some way from those that were captured, and therefore the results might not be generalizable to those intubations.

The first pass success rate of $65.8 \%$ in the supine group in our study is lower than reported first past success rates in other studies, which generally range from 70-90\% [20-22]. This may suggest that there are factors that we did not capture that made the intubations in 
our supine group more difficult. However, this difference could also be related to methods of data collection. Most previous studies reporting EM resident success rates rely on self-reporting of number of attempts by the intubator. We used a strict definition of attempt with frequent reminders to observers and two observers per case in an effort to make sure all attempts were counted. The fact that there were 7 discrepancies in our data set and in 5 of these cases it was determined that there were multiple attempts suggests that in general attempts may be underreported in intubation studies. Use of video recording and review by independent observers may provide more accurate data in future studies.

These limitations prevent us from conclusively attributing the differences in first pass success to patient position. Nevertheless, our study demonstrates that upright intubation is feasible in the ED with high rates of success and intubator satisfaction. Combined with emerging literature suggesting this technique is associated with improved glottic views and decreased complications, it indicates that this is a topic that warrants further study. In particular, a randomized control trial would help limit selection bias.

Other factors that were associated with an increased likelihood of first pass success in our study include increased resident experience level and patient gender. The association between male gender and increased difficulty of intubation has been previously reported [2325], but is underexplored in the literature. Further investigation into gender-related differences would also be helpful.

\section{Conclusions}

Our study adds to a growing body of evidence that there are advantages to performing endotracheal intubation in an upright rather than the traditional supine position. To our knowledge, this is the first prospective study examining success rates of upright endotracheal intubation in the ED. High success rates with upright positioning suggest this is an area that deserves further study. A randomized control trial would be the next step to more conclusively measure the benefits of upright intubation. 


\section{$\underline{\text { References }}$}

[1] Szmuk P, Ezri T, Evron S, Roth Y, Katz J. A brief history of tracheostomy and tracheal intubation, from the Bronze Age to the Space Age. Intensive care medicine. Feb 2008;34(2):222-228.

[2] Mouton WG, Bessell JR, Maddern GJ. Looking back to the advent of modern endoscopy: 150th birthday of Maximilian Nitze. World journal of surgery. Dec 1998;22(12):1256-1258.

[3] Burkle CM, Zepeda FA, Bacon DR, Rose SH. A historical perspective on use of the laryngoscope as a tool in anesthesiology. Anesthesiology. Apr 2004;100(4):1003-1006.

[4] Mort TC. Emergency tracheal intubation: complications associated with repeated laryngoscopic attempts. Anesthesia and analgesia. Aug 2004;99(2):607-613, table of contents.

[5] Schwartz DE, Matthay MA, Cohen NH. Death and other complications of emergency airway management in critically ill adults. A prospective investigation of 297 tracheal intubations. Anesthesiology. Feb 1995;82(2):367-376.

[6] Jaber S, Amraoui J, Lefrant JY, et al. Clinical practice and risk factors for immediate complications of endotracheal intubation in the intensive care unit: a prospective, multiple-center study. Critical care medicine. Sep 2006;34(9):2355-2361.

[7] Li J, Murphy-Lavoie H, Bugas C, Martinez J, Preston C. Complications of emergency intubation with and without paralysis. The American journal of emergency medicine. Mar 1999;17(2):141-143.

[8] Mort TC. Complications of emergency tracheal intubation: hemodynamic alterations--part I. Journal of intensive care medicine. May-Jun 2007;22(3):157-165.

[9] Mort TC. Complications of emergency tracheal intubation: immediate airway-related consequences: part II. Journal of intensive care medicine. Jul-Aug 2007;22(4):208-215.

[10]Griesdale DE, Bosma TL, Kurth T, Isac G, Chittock DR. Complications of endotracheal intubation in the critically ill. Intensive care medicine. Oct 2008;34(10):1835-1842.

[11]Dargin JM, Emlet LL, Guyette FX. The effect of body mass index on intubation success rates and complications during emergency airway management. Internal and emergency medicine. Feb 2013;8(1):75-82. 
[12]Jaber S, Jung B, Corne $\mathrm{P}$, et al. An intervention to decrease complications related to endotracheal intubation in the intensive care unit: a prospective, multiple-center study. Intensive care medicine. Feb 2010;36(2):248-255.

[13]Kim GW, Koh Y, Lim CM, Han M, An J, Hong SB. Does medical emergency team intervention reduce the prevalence of emergency endotracheal intubation complications? Yonsei medical journal. Jan 2014;55(1):92-98.

[14]Khandelwal N, Khorsand S, Mitchell SH, Joffe AM. Head-elevated patient positioning decreases complications of emergent tracheal intubation in the ward and intensive care unit. Anesthesia and analgesia. Apr 2016;122(4):1101-1107.

[15]Lane S, Saunders D, Schofield A, Padmanabhan R, Hildreth A, Laws D. A prospective, randomised controlled trial comparing the efficacy of pre-oxygenation in the 20 degrees head-up vs supine position. Anaesthesia. Nov 2005;60(11):1064-1067.

[16]Dixon BJ, Dixon JB, Carden JR, et al. Preoxygenation is more effective in the 25 degrees head-up position than in the supine position in severely obese patients: a randomized controlled study. Anesthesiology. Jun 2005;102(6):1110-1115; discussion 1115A.

[17]Ramkumar V, Umesh G, Philip FA. Preoxygenation with 20 masculine head-up tilt provides longer duration of non-hypoxic apnea than conventional preoxygenation in non-obese healthy adults. Journal of anesthesia. Apr 2011;25(2):189-194.

[18]Lee BJ, Kang JM, Kim DO. Laryngeal exposure during laryngoscopy is better in the 25 degrees backup position than in the supine position. British journal of anaesthesia. Oct 2007;99(4):581-586.

[19]Turner JS, Ellender TJ, Okonkwo ER et al. Cross-over study of novice intubators performing endotracheal intubation in an upright versus supine position. Intern Emerg Med (2016). doi:10.1007/s11739-016-1481-z

[20]Sakles JC, Chiu S, Mosier J, et al. The importance of first pass success when performing orotracheal intubation in the emergency department. Acad Emerg Med. 2013 Jan;20(1):71-8.

[21]Sagarin MJ, Barton ED, Chng YM, Walls RM, and NEAR Investigators. Airway management by US and Canadian emergency medicine residents: a multicenter analysis of more than 6,000 endotracheal intubation attempts. Ann Emerg Med. 2005 Oct;46(4):328-36

[22]Brown CA $3^{\text {rd }}$, Bair AE, Pallin DJ, Walls RM, and NEAR Investigators. Techniques, success, and adverse events of emergency department adult intubations. Ann Emerg Med. 2015 Apr;65(4):363-370

[23]Diggs LA, Viswakula SD, Sheth-Chandra M, et al. A pilot model for predicting the success of prehospital endotracheal intubation. Am J Emerg Med. 2015 Feb;33(2):202-8.

[24]Neligan PJ, Porter S, Max B, Malhotra G, Greenblatt EP, Ochroch EA. Obstructive sleep apnea is not a risk factor for difficult intubation in morbidly obese patients. Anesth Analg. 2009 Oct;109(4):1182-6. 
[25]Tremblay $\mathrm{MH}$, Williams S, Robitaille A, Drolet P. Poor visualization during direct laryngoscopy and high upper lip bite test score are predictors of difficult intubation with the GlideScope videolaryngoscope. Anesth Analg. 2008 May;106(5):1495-500 